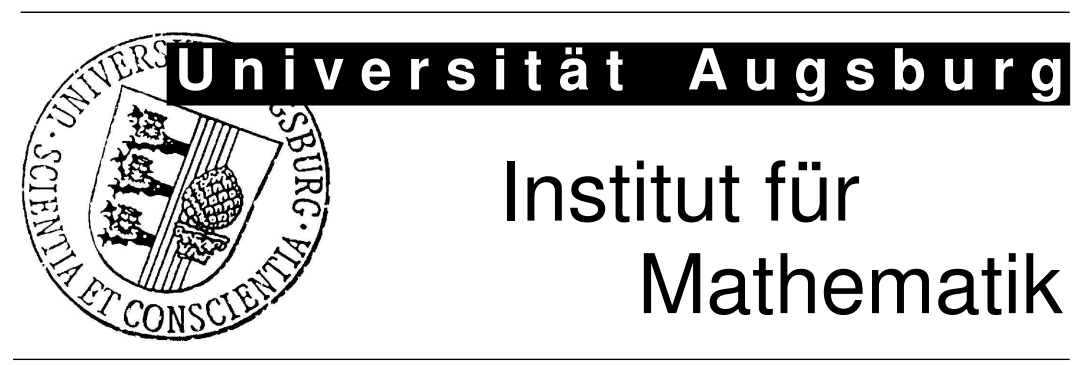

Harbir Antil, Ronald H.W. Hoppe, Christopher Linsenmann

Path-Following Primal-Dual Interior-Point Methods for Shape Optimization of Stationary Flow Problems 


\section{Impressum:}

Herausgeber:

Institut für Mathematik

Universität Augsburg

86135 Augsburg

http://www . math. uni-augsburg.de/forschung/preprint/

ViSdP:

Ronald H.W. Hoppe

Institut für Mathematik

Universität Augsburg

86135 Augsburg

Preprint: Sämtliche Rechte verbleiben den Autoren (C) 2007 


\title{
Path-following primal-dual interior-point methods for shape optimization of stationary flow problems
}

\author{
H. Antil ${ }^{1}$, R.H.W. Hoppe ${ }^{1,2}$, and Chr. Linsenmann ${ }^{1,2}$ \\ ${ }^{1}$ Department of Mathematics, University of Houston, U.S.A. \\ ${ }^{2}$ Institute of Mathematics, University of Augsburg, Germany
}

\begin{abstract}
We consider shape optimization of Stokes flow in channels where the objective is to design the lateral walls of the channel in such a way that a desired velocity profile is achieved. This amounts to the solution of a PDE constrained optimization problem with the state equation given by the Stokes system and the design variables being the control points of a Bézier curve representation of the lateral walls subject to bilateral constraints. Using a finite element discretization of the problem by Taylor-Hood elements, the shape optimization problem is solved numerically by a path-following primal-dual interior-point method applied to the parameter dependent nonlinear system representing the optimality conditions. The method is an all-at-once approach featuring an adaptive choice of the continuation parameter, inexact Newton solves by means of right-transforming iterations, and a monotonicity test for convergence monitoring. The performance of the adaptive continuation process is illustrated by several numerical examples.
\end{abstract}

Keywords: PDE constrained optimization, shape optimization, Stokes flow, primal-dual interiorpoint methods, central path, continuation methods

\section{Introduction}

Structural optimization problems with constraints given by the Stokes equations can be written as follows

$$
\begin{aligned}
& \operatorname{minimize} \quad J(\mathbf{u}, p, \alpha) \\
& \text { over }(\mathbf{u}, p, \alpha) \in \mathbf{V} \times Q \times K \\
& \text { subject to: } \quad S(\mathbf{u}, p, \alpha)=\mathbf{g} .
\end{aligned}
$$

Here, $J: \mathbf{V} \times Q \times U \rightarrow \mathbb{R}$ denotes the objective functional which depends on the state variables $\mathbf{u}$ and $p$ (the velocity and the pressure) in the state space $\mathbf{V} \times Q$ and the design variables $\alpha$ in the design space $U$. The equation (1.1b) corresponds to the Stokes equations, whereas $K \subset U$ refers to the set of admissible design variables.

We note that simplified problems in structural optimization have already been addressed by Bernoulli, Euler, Lagrange and Saint-Venant. However, it became its own discipline during the second half of the last century when the rapidly growing performance of computing platforms and the simultaneously achieved significant improvement of algorithmic tools enabled the appropriate treatment of complex problems (cf. [1, 2, 3, 13, 14, 25, 24, 28, 33, 35, 36, 39] and the references therein). The design criteria in structural optimization are determined by a goal oriented operational behavior of the devices and systems under consideration and typically occur as a nonlinear, often non convex, objective functionals which depend on the state variables describing the operational mode and the design variables determining the shape and the topology. The state variables have to satisfy differential equations or systems

\footnotetext{
${ }^{1}$ The first author acknowledges support by the Texas Learning and Computation Center TLC ${ }^{2}$. The second and third authors acknowledge support by the NSF under Grant No. DMS-0411403 and Grant No. DMS-0511611 as well as by the DFG within the Priority Program SPP 1253'PDE Constrained Optimization'.
} 
thereof representing the underlying physical laws. Technological aspects are taken into account by constraints on the state and/or design variables which may occur both as equality and inequality constraints in the model.

The discretization of such structural optimization problems typically gives rise to equality and inequality constrained nonlinear programming problems. If Newton's method is applied to the KKT conditions, each Newton step requires the solution of a linear algebraic system representing the optimality conditions of a related quadratic programming (QP) problem. Hence, Newton methods can be interpreted in the framework of sequential quadratic programming (SQP) which is the most successful method for solving constrained nonlinear optimization problems $[4,5,6,7,8,9,21,22,34]$. As far as the appropriate treatment of the inequality constraints is concerned, a local optimum can be approximated from within the feasible set, which is the idea behind interior-point methods. The so-called interior-point revolution in continuous optimization started in the eighties of the last century with Karmarkar's polynomial-time linear programming algorithm. It was immediately found that there is a close relationship to barrier functions which had been used long time before for inequality constrained nonlinear programming problems. Nowadays, interior-point methods are well established tools for constrained nonlinear optimization problems (cf., e.g., $[12,15,17,18,19,20,26,27,29,30,31,32,40,41,43,44,45])$. In terms of the barrier parameter, we are faced with a parameter dependent nonlinear system whose solution is referred to as the central path. For the parameter-dependent nonlinear system, continuation methods along the central path are the methods of choice. Such continuation methods have been studied extensively in the literature. A good reference both for theoretical and algorithmic aspects is the recent textbook [16]. The most widely used techniques are path-following predictor-corrector strategies.

In this paper, we consider path-following primal-dual interior-point methods for the shape optimization of stationary flow problems as described by the Stokes system. In particular, we consider Stokes flow in channels where the objective is to design the lateral walls in such a way that a desired velocity profile is obtained. The design variables are chosen as the control points of a Bézier curve representation of the lateral walls. The discretization of the problem is done by Taylor-Hood elements with respect to a shape regular family of simplicial triangulations of the computational domain. The resulting optimality conditions for the discretized problem are solved by an adaptive continuation method in the spirit of [16] applied to the parameter dependent nonlinear system representing the central path. For the computation of the derivatives occurring in the KKT conditions and the Hessian, we use automatic differentiation [23].

The paper is organized as follows: In section 2, we formulate the shape optimization problem along with its finite element discretization and set up the optimality conditions. Section 3 is devoted to the primal-dual approach featuring parameterized minimization subproblems in terms of logarithmic barrier functions that take care of the bilateral constraints on the design variables. The optimality conditions lead to a parameter dependent nonlinear system whose solution is known as the central path. This system is solved by a path-following predictor-corrector type continuation method with an adaptive choice of the continuation parameter (barrier parameter) and a monotonicity test for convergence monitoring. At each continuation step, the Newton increments are computed by means of an iterative scheme based on right-transforming iterations whose construction essentially takes advantage of the specific structure of the associated Hessian. The final section 4 contains a detailed documentation of numerical examples illustrating the performance of the adaptive continuation method. 


\section{The shape optimization problem}

\subsection{Setting of the problem}

We consider Stokes flow in a channel occupying bounded domain $\Omega(\alpha) \subset \mathbb{R}^{2}$ with boundary $\Gamma(\alpha)=\Gamma_{\text {in }}(\alpha) \cup \Gamma_{\text {lat }}(\alpha) \cup \Gamma_{\text {out }}(\alpha)$, where $\Gamma_{\text {lat }}(\alpha):=\Gamma_{\text {bot }}(\alpha) \cup \Gamma_{\text {top }}(\alpha)$, depending on a design variable $\alpha=\left(\alpha_{1}, \cdots, \alpha_{m}\right)^{T} \in \mathbb{R}^{m}$ (cf. Fig. 1 ).

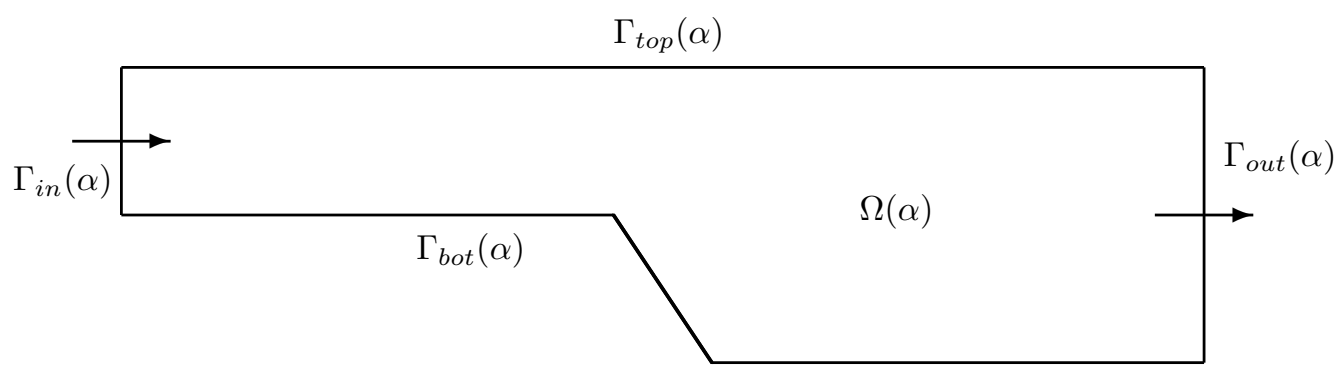

Fig. 1: Stokes flow in a channel with a backward facing step

Denoting the velocity and the pressure by $\mathbf{u}:=\left(u_{1}, u_{2}\right)^{T}$ and $p$, respectively, and given a velocity and pressure profiles $\mathbf{u}^{\mathrm{d}}, p^{d}$, the objective is to design the geometry of the domain in such a way that $\mathbf{u}^{\mathbf{d}}, p^{d}$ are attained as closely as possible. This amounts to the minimization problem

$$
\inf _{\mathbf{u}, p, \alpha} J(\mathbf{u}, p, \alpha) \quad, \quad J(\mathbf{u}, p, \alpha):=\frac{\lambda_{1}}{2} \int_{\Omega(\alpha)}\left|\mathbf{u}-\mathbf{u}^{\mathbf{d}}\right|^{2} d x+\frac{\lambda_{2}}{2} \int_{\Omega(\alpha)}\left|p-p^{d}\right|^{2} d x
$$

subject to the PDE constraint (Stokes flow)

$$
\begin{aligned}
& -\nu \Delta \mathbf{u}+\nabla p=\mathbf{f} \text { in } \Omega(\alpha), \\
& \nabla \cdot \mathbf{u} \quad=0 \text { in } \Omega(\alpha) \text {, } \\
& \mathbf{n} \cdot \mathbf{u}=\left\{\begin{array}{c}
u_{\text {in }} \text { on } \Gamma_{\text {in }}(\alpha) \\
u_{\text {out }} \text { on } \Gamma_{\text {out }}(\alpha) \\
0 \text { on } \Gamma_{\text {lat }}(\alpha)
\end{array},\right. \\
& \mathbf{t} \cdot \mathbf{u}=0 \text { on } \Gamma(\alpha),
\end{aligned}
$$

and subject to the bilateral constraints on the design variable $\alpha$

$$
\alpha \in K:=\left\{\alpha_{i} \mid \alpha_{i}^{\min } \leq \alpha_{i} \leq \alpha_{i}^{\max }, 1 \leq i \leq m\right\},
$$

where $\lambda_{i} \geq 0,1 \leq i \leq 2, \lambda_{1}+\lambda_{2}>0$, are weighting factors, $\nu$ is the viscosity, $\mathbf{f}$ refers to a forcing term, $\mathbf{n}, \mathbf{t}$ denote the unit outward normal and unit tangential vector on $\Gamma(\alpha), u_{i n}, u_{\text {out }}$ stand for a inflow/outflow profiles satisfying the compatibility condition $\int_{\Gamma_{\text {in }}(\alpha)} u_{\text {in }} d s=\int_{\Gamma_{\text {out }}(\alpha)} u_{\text {out }} d s$, and $\alpha^{\text {min }}, \alpha^{\max } \in \mathbb{R}^{m}$. Note that $u_{\text {out }}:=\mathbf{n} \cdot \mathbf{u}$ on $\Gamma_{\text {out }}(\alpha)$ is then determined by means of $(2.2 \mathrm{~b})$. The state equations $(2.2 \mathrm{a})-(2.2 \mathrm{~d})$ have to be understood in a weak sense. We adopt standard notation from Lebesgue and Sobolev space theory. In particular, we set

$$
\mathbf{V}:=\left\{\mathbf{v} \in H^{1}(\Omega(\alpha))^{2} \mid \mathbf{v} \text { satisfies }(2.2 \mathrm{c}),(2.2 \mathrm{~d})\right\} \quad, \quad Q:=L_{0}^{2}(\Omega(\alpha)) .
$$


We denote by $\mathbf{V}^{*}$ and $Q^{*}$ the duals of $\mathbf{V}$ and $Q$, and we introduce $A(\alpha): \mathbf{V} \rightarrow \mathbf{V}^{*}$ and $B(\alpha): \mathbf{V} \rightarrow Q^{*}$ as the operators associated with the bilinear forms

$$
\begin{gathered}
a(\mathbf{v}, \mathbf{w} ; \alpha)):=\nu \int_{\Omega(\alpha)} \nabla \mathbf{v} \cdot \nabla \mathbf{w} d x \quad, \quad \mathbf{v} \in \mathbf{V}, \mathbf{w} \in H_{0}^{1}(\Omega(\alpha))^{2}, \\
b(\mathbf{v}, q ; \alpha):=-\int_{\Omega(\alpha)} \nabla \cdot \mathbf{v} q d x \quad, \quad \mathbf{v} \in \mathbf{V}, q \in L^{2}(\Omega(\alpha)) .
\end{gathered}
$$

We further introduce the state space $\mathbf{Y}:=\mathbf{V} \times Q$ and the operator $S: \mathbf{Y} \times K \rightarrow \mathbf{Y}^{*}$ according to

$$
S(\mathbf{y}, \alpha):=\left(\begin{array}{cc}
A(\alpha) & B^{*}(\alpha) \\
B(\alpha) & 0
\end{array}\right)\left(\begin{array}{l}
\mathbf{u} \\
p
\end{array}\right) \quad, \quad \mathbf{y}=(\mathbf{u}, p)^{T} \in Y, \alpha \in K .
$$

We note that $S(\cdot, \alpha): \mathbf{Y} \rightarrow \mathbf{Y}^{*}, \alpha \in K$, is the Stokes operator. Then, the state equations (2.2a)-(2.2d) can be written in operator form according to

$$
S(\mathbf{y}, \alpha)=\mathbf{g} .
$$

where $\mathbf{g}:=(\mathbf{f}, 0)^{T}$. The design variables $\alpha_{i}, 1 \leq i \leq m$, are chosen as the Bézier control points in a Bézier representation of $\Gamma(\alpha)$ as a globally continuous composition of polynomial curve segments of some polynomial degree. We choose $\hat{\alpha} \in K$ as a reference design and refer to $\hat{\Omega}:=\Omega(\hat{\alpha})$ as the associated reference domain. Then, the actual domain $\Omega(\alpha)$ can be obtained from the reference domain $\hat{\Omega}$ by means of an isomorphism

$$
\begin{aligned}
\Omega(\alpha) & =\Phi(\hat{\Omega} ; \alpha), \\
\Phi(\hat{x} ; \alpha) & =\left(\Phi_{1}(\hat{x} ; \alpha), \Phi_{2}(\hat{x} ; \alpha)\right)^{T}, \hat{x}=\left(\hat{x}_{1}, \hat{x}_{2}\right)^{T}
\end{aligned}
$$

with continuous components $\Phi_{i}, 1 \leq i \leq 2$. We assume:

(A): The functions $\Phi_{i}, 1 \leq i \leq 2$, are subdifferentiable in $\hat{x} \in \hat{\Omega}$ with uniformly bounded subdifferentials $\partial \Phi_{i}$ and twice continuously differentiable in $\alpha_{i}, 1 \leq i \leq m$.

The advantage of using the reference domain $\hat{\Omega}$ is that finite element approximations of (2.1) can be performed with respect to that fixed domain without being forced to remesh.

We denote by $\left(\mathcal{T}_{h}(\hat{\Omega})\right)_{\mathbb{N}}$ a shape regular family of simplicial triangulations of $\hat{\Omega}$. By means of $(2.7)$, these triangulations induce an associated family $\left(\mathcal{T}_{h}(\Omega(\alpha))\right)_{\mathbb{N}}$ of simplicial triangulations of the actual physical domains $\Omega(\alpha)$.

We use Taylor-Hood P2/P1 elements (cf., e.g., [11]) for the discretization of the velocity $\mathbf{u} \in \mathbf{V}$ and the pressure $p \in Q$ denoting the associated trial spaces by $\mathbf{V}_{h}$ and $Q_{h}$ with $\operatorname{dim} \mathbf{V}_{h}=n_{1}$ and $\operatorname{dim} Q_{h}=n_{2}$, respectively. This gives rise to an objective functional $J_{h}: \mathbb{R}^{n} \times \mathbb{R}^{m}, n:=n_{1}+n_{2}$, by means of

$$
J_{h}\left(\mathbf{u}_{h}, p_{h}, \alpha\right):=\frac{\lambda_{1}}{2}\left(\mathbf{u}_{h}-\mathbf{u}_{h}^{d}\right)^{T} I_{1, h}(\alpha)\left(\mathbf{u}_{h}-\mathbf{u}_{h}^{d}\right)+\frac{\lambda_{2}}{2} p_{h}^{T} I_{2, h}(\alpha) p_{h},
$$

where $I_{\nu, h}(\alpha), 1 \leq \nu \leq 2$, are the associated mass matrices and $\mathbf{u}_{h}^{d} \in \mathbb{R}^{n_{1}}, p_{h} \in \mathbb{R}^{n_{2}}$ result from the $L^{2}$-projections of $\mathbf{u}^{d}, p^{d}$ onto $\mathbf{V}_{h}$ and $Q_{h}$, respectively. The discretized shape optimization problem can be stated as

$$
\inf _{\mathbf{u}_{h}, p_{h}, \alpha} J_{h}\left(\mathbf{u}_{h}, p_{h}, \alpha\right)
$$

subject to the algebraic saddle point problem

$$
S_{h}\left(\mathbf{y}_{h}, \alpha\right):=\left(\begin{array}{cc}
A_{h}(\alpha) & B_{h}^{T}(\alpha) \\
B_{h}(\alpha) & 0
\end{array}\right)\left(\begin{array}{l}
\mathbf{u}_{h} \\
p_{h}
\end{array}\right)=\left(\begin{array}{l}
\mathbf{g}_{1, h} \\
\mathbf{g}_{2, h}
\end{array}\right),
$$


representing the Taylor-Hood approximation of the Stokes system (2.5).

Due to the dependence of the domain on the design parameters $\alpha_{i}, 1 \leq i \leq m$, the objective functional $J_{h}$ is nonconvex. Therefore, there may exist a multitude of local minima. Throughout the following, we assume that $\left(\mathbf{y}_{h}^{*}, \alpha^{*}\right) \in \mathbb{R}^{n} \times K$ is a strict local solution of (2.9), i.e., there exists a neighborhood $\mathcal{U}\left(\mathbf{y}_{h}^{*}, \alpha^{*}\right) \subset \mathbb{R}^{n} \times K$ such that

$$
J_{h}\left(\mathbf{y}_{h}^{*}, \alpha^{*}\right)<J\left(\mathbf{y}_{h}, \alpha\right) \quad, \quad\left(\mathbf{y}_{h}, \alpha\right) \in \mathcal{U}\left(\mathbf{y}_{h}^{*}, \alpha^{*}\right) \backslash\left\{\left(\mathbf{y}_{h}^{*}, \alpha^{*}\right)\right\} .
$$

For notational convenience, in the sequel we will drop the discretization index $h$.

\subsection{Optimality conditions}

We introduce Lagrange multipliers $\boldsymbol{\lambda}:=\left(\boldsymbol{\lambda}_{\mathbf{u}}, \lambda_{p}\right)^{T} \in \mathbb{R}^{n_{1}} \times \mathbb{R}^{n_{2}}$ and $\sigma:=\left(\sigma_{1}, \sigma_{2}\right)^{T}, \sigma_{k} \in$ $\mathbb{R}_{+}^{m}, 1 \leq k \leq 2$. The saddle point formulation of the minimization problem (2.9) is

$$
\inf _{\mathbf{y}, \alpha} \sup _{\boldsymbol{\lambda}, \sigma} L(\mathbf{y}, \alpha, \boldsymbol{\lambda}, \sigma) .
$$

Here, the Lagrangian $L$ is given by

$$
L(\mathbf{y}, \alpha, \boldsymbol{\lambda}, \sigma):=J(\mathbf{u}, p, \alpha)+\boldsymbol{\lambda}^{T}(S(\mathbf{y}, \alpha)-\mathbf{g})+\sigma_{1}^{T}\left(\alpha^{\min }-\alpha\right)+\sigma_{2}^{T}\left(\alpha-\alpha^{\max }\right) .
$$

Denoting by $\mathbf{x}:=(\mathbf{y}, \alpha)^{T}$ the primal variables, the first order necessary optimality conditions are given by

$$
\begin{aligned}
L_{\mathbf{y}}(\mathbf{x}, \boldsymbol{\lambda}, \boldsymbol{\sigma}) & =0, \\
L_{\alpha}(\mathbf{x}, \boldsymbol{\lambda}, \boldsymbol{\sigma}) & =0, \\
L_{\boldsymbol{\lambda}}(\mathbf{x}, \boldsymbol{\lambda}, \boldsymbol{\sigma}) & =0, \\
L_{\sigma_{1}}(\mathbf{x}, \boldsymbol{\lambda}, \boldsymbol{\sigma})=\alpha^{\min }-\alpha & \leq 0, \\
\left(\sigma_{1}\right)^{T}\left(\alpha^{\min }-\alpha\right) & =0, \\
L_{\sigma_{2}}(\mathbf{x}, \boldsymbol{\lambda}, \boldsymbol{\sigma})=\alpha-\alpha^{\max } & \leq 0, \\
\left(\sigma_{2}\right)^{T}\left(\alpha-\alpha^{\max }\right) & =0 .
\end{aligned}
$$

In particular, we find

$$
\begin{aligned}
L_{\mathbf{u}}(\cdots)= & \lambda_{1} I_{1}(\alpha)\left(\mathbf{u}-\mathbf{u}^{d}\right)+A(\alpha) \boldsymbol{\lambda}_{\mathbf{u}}+B^{T}(\alpha) \lambda_{p}=0 \\
L_{p}(\cdots)= & \lambda_{2} I_{2}(\alpha) p+B(\alpha) \boldsymbol{\lambda}_{\mathbf{u}}=0 \\
L_{\alpha}(\cdots)= & \nabla_{\alpha} J(\mathbf{u}, p, \alpha)+\nabla_{\alpha}\left\langle A(\alpha) \mathbf{u}+B^{T}(\alpha) p-\mathbf{f}, \boldsymbol{\lambda}_{\mathbf{u}}\right\rangle+ \\
& \quad+\nabla_{\alpha}\left\langle B(\alpha) \mathbf{u}, \lambda_{p}\right\rangle-\sigma_{1}+\sigma_{2}=0 \\
L_{\boldsymbol{\lambda}_{\mathbf{u}}}(\cdots)= & A(\alpha) \mathbf{u}+B^{T}(\alpha) p-\mathbf{f}=0 \\
L_{\lambda_{p}}(\cdots)= & B(\alpha) \mathbf{u}=0 .
\end{aligned}
$$

Theorem 1. Let $\left(\mathbf{y}^{*}, \alpha^{*}\right) \in \mathbb{R}^{n} \times K$ be a strict local solution of (2.9) and assume that the constraints are qualified in the sense of the linear independence constraint qualification (cf., e.g, [10]). Then, there exist multipliers $\boldsymbol{\lambda}^{*}=\left(\boldsymbol{\lambda}_{\mathbf{u}}^{*}, \lambda_{p}^{*}\right) \in \mathbb{R}^{n}$ and $\sigma^{*}=\left(\sigma_{1}^{*}, \sigma_{2}^{*}\right), \sigma_{k}^{*} \in \mathbb{R}_{+}^{m}, 1 \leq$ $k \leq 2$, such that $\left(\mathbf{y}^{*}, \alpha^{*}, \boldsymbol{\lambda}^{*}, \sigma^{*}\right)$ satisfies the KKT system $(2.14 \mathrm{a})-(2.14 \mathrm{e})$.

Proof. The proof follows from standard results of optimization theory (cf., e.g., [10]).

For the second order derivatives of the Lagrangian $L$ with respect to the primal variables $\mathbf{y}$ we obtain

$$
\begin{array}{cll}
L_{\mathbf{u u}}(\cdots)=\lambda_{1} I_{1}(\alpha) & , \quad L_{\mathbf{u} p}(\cdots)=0 \\
L_{p \mathbf{u}}(\cdots)=0, & L_{p p}(\cdots)=\lambda_{2} I_{2}(\alpha) .
\end{array}
$$


Consequently, the Hessian $L_{\mathbf{x x}}$ has the form

$$
L_{\mathbf{x x}}(\cdots)=\left(\begin{array}{cc}
I(\alpha) & L_{\mathbf{y} \alpha} \\
L_{\alpha \mathbf{y}} & L_{\alpha \alpha}
\end{array}\right),
$$

where

$$
\begin{aligned}
& I(\alpha)=\left(\begin{array}{cc}
\lambda_{1} I_{1}(\alpha) & 0 \\
0 & \lambda_{2} I_{2}(\alpha)
\end{array}\right), \\
& L_{\alpha \mathbf{y}}=\left(L_{\alpha \mathbf{u}} L_{\alpha p}\right) \quad, \quad L_{\mathbf{y} \alpha}=\left(L_{\mathbf{u} \alpha} L_{p \alpha}\right)^{T} .
\end{aligned}
$$

Note that

$$
\begin{aligned}
& L_{\mathbf{u} \alpha}: \mathbb{R}^{m} \rightarrow \mathbb{R}^{n_{1}} \quad, \quad L_{\alpha \mathbf{u}}: \mathbb{R}^{n_{1}} \rightarrow \mathbb{R}^{m}, \\
& L_{p \alpha}: \mathbb{R}^{m} \rightarrow \mathbb{R}^{n_{2}}, \quad L_{\alpha p}: \mathbb{R}^{n_{2}} \rightarrow \mathbb{R}^{m}, \\
& L_{\mathbf{u} \alpha}=L_{\alpha \mathbf{u}}^{*}, L_{p \alpha}=L_{\alpha p}^{*},
\end{aligned}
$$

so that $L_{\mathbf{x x}}(\cdots)$ is symmetric. The second order sufficient optimality requires positive definiteness of $L_{\mathbf{x x}}(\cdots)$ at optimality:

Theorem 2. Let $\left(\mathbf{x}^{*}, \boldsymbol{\lambda}^{*}, \sigma^{*}\right) \in \mathbb{R}^{n} \times K \times \mathbb{R}^{n} \times\left(\mathbb{R}_{+}^{m}\right)^{2}$ satisfy the KKT conditions (2.14a)(2.14e) and suppose that $L_{\mathbf{x} \mathbf{x}}\left(\mathbf{x}^{*}, \boldsymbol{\lambda}^{*}, \sigma^{*}\right)$ is positive definite, i.e.,

$$
\mathbf{w}^{T} L_{\mathbf{x x}}\left(\mathbf{x}^{*}, \boldsymbol{\lambda}^{*}, \sigma^{*}\right) \mathbf{w}>0 \quad, \quad \mathbf{w} \in \mathbb{R}^{n} \times K \backslash\{\mathbf{0}\} .
$$

Then, $\left(\mathbf{x}^{*}, \boldsymbol{\lambda}^{*}, \sigma^{*}\right)$ is a strict local solution of (2.1).

Proof. We refer to [10].

\section{The primal-dual interior-point approach}

\subsection{The central path}

We couple the inequality constraints $(2.3)$ by logarithmic barrier functions with a barrier parameter $\beta=1 / \mu>0, \mu \rightarrow \infty$, resulting in the following parameterized family of minimization subproblems

$$
\inf _{\mathbf{y}, \alpha} B^{(\mu)}(\mathbf{y}, \alpha)
$$

subject to $(2.6)$, where

$$
B^{(\mu)}(\mathbf{y}, \alpha):=J(\mathbf{y}, \alpha)-\frac{1}{\mu} \sum_{i=1}^{m}\left[\ln \left(\alpha_{i}-\alpha_{i}^{\min }\right)+\ln \left(\alpha_{i}^{\max }-\alpha_{i}\right)\right] .
$$

The dual aspect is to couple the PDE constraint (2.6) by a Lagrange multiplier $\boldsymbol{\lambda}=$ $\left(\boldsymbol{\lambda}_{\mathbf{u}}, \lambda_{p}\right)^{T} \in \mathbf{V} \times Q$ which gives rise to the saddle point problem

$$
\inf _{\mathbf{y}, \alpha} \sup _{\boldsymbol{\lambda}} L^{(\mu)}(\mathbf{y}, \boldsymbol{\lambda}, \alpha)
$$

where the Lagrangian $L^{(\mu)}$ is given by

$$
L^{(\mu)}(\mathbf{y}, \boldsymbol{\lambda}, \alpha)=B^{(\mu)}(\mathbf{y}, \alpha)+\langle S(\mathbf{y}, \alpha)-\mathbf{g}, \boldsymbol{\lambda}\rangle .
$$

The central path $\mu \longmapsto x(\mu):=(\mathbf{y}(\mu), \boldsymbol{\lambda}(\mu), \alpha(\mu))^{T}$ is given as the solution of the nonlinear system

$$
F(x(\mu), \mu)=\left(\begin{array}{c}
L_{\mathbf{y}}^{(\mu)}(\mathbf{y}, \boldsymbol{\lambda}, \alpha) \\
L_{\lambda}^{(\mu)}(\mathbf{y}, \boldsymbol{\lambda}, \alpha) \\
L_{\alpha}^{(\mu)}(\mathbf{y}, \boldsymbol{\lambda}, \alpha)
\end{array}\right)=0
$$


which represents the first order necessary optimality conditions for (3.1).

Setting $\mathbf{y}^{d}:=\left(\mathbf{u}^{d}, p^{d}\right)$, the derivatives of the Lagrangian are given by

$$
\begin{aligned}
& L_{\mathbf{y}}^{(\mu)}(\mathbf{y}, \boldsymbol{\lambda}, \alpha)=I(\alpha)\left(\mathbf{y}-\mathbf{y}^{d}\right)+S(\boldsymbol{\lambda}, \alpha) \\
& L_{\boldsymbol{\lambda}}^{(\mu)}(\mathbf{y}, \boldsymbol{\lambda}, \alpha)=S(\mathbf{y}, \alpha)-\mathbf{g} \\
& L_{\alpha_{i}}^{(\mu)}(\mathbf{y}, \boldsymbol{\lambda}, \alpha)=\partial_{\alpha_{i}} J(\mathbf{u}, p)+<\partial_{\alpha_{i}} S(\mathbf{y}, \alpha), \boldsymbol{\lambda}>-\frac{1}{\mu\left(\alpha_{i}-\alpha_{i}^{\min }\right)}+\frac{1}{\mu\left(\alpha_{i}^{\max }-\alpha_{i}\right)} .
\end{aligned}
$$

Theorem 3. Let $\left(\mathbf{y}^{*}, \alpha^{*}, \boldsymbol{\lambda}^{*}, \sigma^{*}\right) \in \mathbf{Y} \times K \times \mathbb{R}^{n} \times\left(\mathbb{R}_{+}^{m}\right)^{2}$ satisfy the first order necessary optimality conditions $(2.14 \mathrm{a})-(2.14 \mathrm{e})$ with strict complementarity in $(2.14 \mathrm{~d}),(2.14 \mathrm{e})$ and the second order sufficient optimality condition (2.20). Moreover, assume that the linear independence constraint qualification holds true. Then, there exists $\mu_{\min }>0$ such that for all $\mu>\mu_{\min }$ the minimization subproblems (3.1) admit unique solutions $(\mathbf{y}(\mu), \boldsymbol{\lambda}(\mu), \alpha(\mu))$ satisfying (3.5) and converging to $\left(\mathbf{y}^{*}, \boldsymbol{\lambda}^{*}, \alpha^{*}\right)$ as $\mu \rightarrow \infty$.

Proof. We refer to [43].

\subsection{Adaptive path-following continuation method}

For the solution of the parameter-dependent nonlinear system (3.5) we use an adaptive path-following predictor-corrector strategy along the lines of [16].

Predictor Step: The predictor step relies on tangent continuation along the trajectory of the Davidenko equation

$$
F_{\mathbf{x}}(\mathbf{x}(\mu), \mu) \mathbf{x}^{\prime}(\mu)=-F_{\mu}(\mathbf{x}(\mu), \mu) .
$$

Given some approximation $\tilde{\mathbf{x}}\left(\mu_{k}\right)$ at $\mu_{k}>0$, compute $\tilde{\mathbf{x}}^{(0)}\left(\mu_{k+1}\right)$, where $\mu_{k+1}=\mu_{k}+\Delta \mu_{k}^{(0)}$, according to

$$
\begin{aligned}
F_{x}\left(\tilde{\mathbf{x}}\left(\mu_{k}\right), \mu_{k}\right) \delta \mathbf{x}\left(\mu_{k}\right) & =-F_{\mu}\left(\tilde{\mathbf{x}}\left(\mu_{k}\right), \mu_{k}\right), \\
\tilde{\mathbf{x}}^{(0)}\left(\mu_{k+1}\right) & =\tilde{\mathbf{x}}\left(\mu_{k}\right)+\Delta \mu_{k}^{(0)} \delta \mathbf{x}\left(\mu_{k}\right) .
\end{aligned}
$$

We use $\Delta \mu_{0}^{(0)}=\Delta \mu_{0}$ for some given initial step size $\Delta \mu_{0}$, whereas for $k \geq 1$ the predicted step size $\Delta \mu_{k}^{(0)}$ is chosen by

$$
\Delta \mu_{k}^{(0)}:=\left(\frac{\left\|\Delta \mathbf{x}^{(0)}\left(\mu_{k}\right)\right\|}{\left\|\tilde{\mathbf{x}}\left(\mu_{k}\right)-\tilde{\mathbf{x}}^{(0)}\left(\mu_{k}\right)\right\|} \frac{\sqrt{2}-1}{2 \Theta\left(\mu_{k}\right)}\right)^{1 / 2} \Delta \mu_{k-1},
$$

where $\Delta \mu_{k-1}$ is the computed continuation step size, $\Delta \mathbf{x}^{(0)}\left(\mu_{k}\right)$ is the first Newton correction (see below), and $\Theta\left(\mu_{k}\right)<1$ is the contraction factor associated with a successful previous continuation step.

Corrector step: As a corrector, we use Newton's method applied to $F\left(\mathbf{x}\left(\mu_{k+1}\right), \mu_{k+1}\right)=0$ with $\tilde{\mathbf{x}}^{(0)}\left(\mu_{k+1}\right)$ from (3.8) as a start vector. In particular, for $\ell \geq 0$ and $j_{\ell} \geq 0$ we compute $\Delta x^{(j \ell)}\left(\mu_{k+1}\right)$ according to

$$
F^{\prime}\left(\tilde{x}^{\left(j_{\ell}\right)}\left(\mu_{k+1}\right), \mu_{k+1}\right) \Delta x^{\left(j_{\ell}\right)}\left(\mu_{k+1}\right)=-F\left(\tilde{x}^{\left(j_{\ell}\right)}\left(\mu_{k+1}\right), \mu_{k+1}\right)
$$

and $\overline{\Delta x}^{\left(j_{\ell}\right)}\left(\mu_{k+1}\right)$ as the associated simplified Newton correction

$$
F^{\prime}\left(\tilde{x}^{\left(j_{\ell}\right)}\left(\mu_{k+1}\right), \mu_{k+1}\right) \overline{\Delta x}^{\left(j_{\ell}\right)}\left(\mu_{k+1}\right)=-F\left(\tilde{x}^{\left(j_{\ell}\right)}\left(\mu_{k+1}\right)+\Delta x^{\left(j_{\ell}\right)}\left(\mu_{k+1}\right), \mu_{k+1}\right) .
$$

We monitor convergence of Newton's method by means of

$$
\Theta^{\left(j_{\ell}\right)}\left(\mu_{k+1}\right):=\left\|\overline{\Delta x}^{\left(j_{\ell}\right)}\left(\mu_{k+1}\right)\right\| /\left\|\Delta x^{\left(j_{\ell}\right)}\left(\mu_{k+1}\right)\right\| .
$$


In case of successful convergence, we accept the current step size and proceed with the next continuation step. However, if the monotonicity test

$$
\Theta^{(j \ell)}\left(\mu_{k+1}\right)<1
$$

fails for some $j_{\ell} \geq 0$, the continuation step has to be repeated with the reduced step size

$$
\Delta \mu_{k}^{(\ell+1)}:=\left(\frac{\sqrt{2}-1}{g\left(\Theta^{\left(j_{\ell}\right)}\right)}\right)^{1 / 2} \Delta \mu_{k}^{(\ell)} \quad, \quad g(\Theta):=\sqrt{\Theta+1}-1
$$

until we either achieve convergence or for some prespecified lower bound $\Delta \mu_{\min }$ observe

$$
\Delta \mu_{k}^{(\ell+1)}<\Delta \mu_{\min }
$$

In the latter case, we stop the algorithm and report convergence failure.

\subsection{Inexact Newton method by right-transforming iterations}

As inner iterations we use an inexact Newton method featuring right-transforming iterations (cf., e.g., $[29,30,31])$. To this end, we introduce the slack variables

$$
z_{i}^{(1)}:=\frac{1}{\mu\left(\alpha_{i}-\alpha_{i}^{\min }\right)} \quad, \quad z_{i}^{(2)}:=\frac{1}{\mu\left(\alpha_{i}^{\max }-\alpha_{i}\right)} \quad, \quad 1 \leq i \leq m
$$

In the slack variable formulation of the KKT system, a Newton step in the increments

$$
\Delta x:=\left(\Delta \mathbf{u}, \Delta p, \Delta \boldsymbol{\lambda}_{\mathbf{u}}, \Delta \lambda_{p}, \Delta \alpha, \Delta z^{(1)}, \Delta z^{(2)}\right)^{T}
$$

reads as follows (for notational convenience, in the following the upper index $(\mu)$ in the Lagrangian will be dropped):

$$
\left(\begin{array}{cc|cc|c|cc}
\lambda_{1} I_{1}(\alpha) & 0 & A(\alpha) & B^{T}(\alpha) & L_{u, \alpha} & 0 & 0 \\
0 & \lambda_{2} I_{2}(\alpha) & B(\alpha) & 0 & L_{p, \alpha} & 0 & 0 \\
-- & -- & -- & -- & -- & -- & -- \\
A(\alpha) & B^{T}(\alpha) & 0 & 0 & L_{\boldsymbol{\lambda}_{\mathbf{u}}, \alpha} & 0 & 0 \\
B(\alpha) & 0 & 0 & 0 & L_{\lambda_{p}, \alpha} & 0 & 0 \\
-- & -- & -- & -- & -- & -- & -- \\
L_{\alpha, u} & L_{\alpha, p} & L_{\alpha, \boldsymbol{\lambda}_{\mathbf{u}}} & L_{\alpha, \lambda_{p}} & L_{\alpha, \alpha} & -I & I \\
-- & -- & -- & -- & -- & -- & -- \\
0 & 0 & 0 & 0 & Z_{1} & D_{1} & 0 \\
0 & 0 & 0 & 0 & -Z_{2} & 0 & D_{2}
\end{array}\right)\left(\begin{array}{c}
\Delta \mathbf{u} \\
\Delta p \\
-- \\
\Delta \boldsymbol{\lambda}_{\mathbf{u}} \\
\Delta \lambda_{p} \\
-- \\
\Delta \alpha \\
-- \\
\Delta z^{(1)} \\
\Delta z^{(2)}
\end{array}\right)=-\mathbf{g}
$$

where

$$
\text { g := }\left(g_{1}, g_{2}, g_{3}, g_{4}, g_{5}, D_{1} Z_{1} e-\mu^{-1} e, D_{2} Z_{2} e-\mu^{-1} e\right)^{T}
$$

with appropriate $g_{i}, 1 \leq i \leq 5, e:=(1, \cdots, 1)^{T}$ and

$$
D_{1}:=\operatorname{diag}\left(\alpha_{i}-\alpha_{i}^{\min }\right), D_{2}:=\operatorname{diag}\left(\alpha_{i}^{\max }-\alpha_{i}\right), Z_{\nu}:=\operatorname{diag}\left(z_{i}^{(\nu)}\right), 1 \leq \nu \leq 2
$$

Since $D_{\nu}, Z_{\nu}, 1 \leq \nu \leq 2$, are diagonal matrices, the slack variables can be easily eliminated 
by condensation (block Gaussian elimination) which leads to the condensed Hessian system

$$
\begin{aligned}
& \left(\begin{array}{cc|cc|c}
\lambda_{1} I_{1}(\alpha) & 0 & A(\alpha) & B^{T}(\alpha) & L_{\mathbf{u}, \alpha} \\
0 & \lambda_{2} I_{2}(\alpha) & \mathbf{B}(\alpha) & 0 & L_{p, \alpha} \\
-- & -- & -- & -- & -- \\
A(\alpha) & B^{T}(\alpha) & 0 & 0 & L_{\boldsymbol{\lambda}_{\mathbf{u}}, \alpha} \\
B(\alpha) & 0 & 0 & 0 & L_{\lambda_{p}, \alpha} \\
-- & -- & -- & -- & -- \\
L_{\alpha, \mathbf{u}} & L_{\alpha, p} & L_{\alpha, \boldsymbol{\lambda}_{\mathbf{u}}} & L_{\alpha, \lambda_{p}} & \tilde{L}_{\alpha, \alpha}
\end{array}\right)\left(\begin{array}{c}
\boldsymbol{\Delta} \mathbf{u} \\
\Delta p \\
-- \\
\Delta \boldsymbol{\lambda}_{\mathbf{u}} \\
\Delta \lambda_{p} \\
-- \\
\Delta \alpha
\end{array}\right)= \\
& =\left(\begin{array}{cc|c}
I(\alpha) & S & L_{\mathbf{v}, \alpha} \\
S & 0 & L_{\boldsymbol{\lambda}, \alpha} \\
-- & -- & -- \\
L_{\alpha, \mathbf{v}} & L_{\alpha, \boldsymbol{\lambda}} & \tilde{L}_{\alpha, \alpha}
\end{array}\right)\left(\begin{array}{c}
\Delta \mathbf{v} \\
\Delta \boldsymbol{\lambda} \\
-- \\
\Delta \alpha
\end{array}\right)=-\left(\begin{array}{c}
\mathbf{h}_{1} \\
\mathbf{h}_{2} \\
-- \\
h_{3}
\end{array}\right)
\end{aligned}
$$

with appropriate $h_{i}, 1 \leq i \leq 3, \mathbf{v}:=(\mathbf{u}, p)^{T}, \boldsymbol{\lambda}:=\left(\boldsymbol{\lambda}_{\mathbf{u}}, \lambda_{p}\right)^{T}$ and

$$
\begin{aligned}
S & :=\left(\begin{array}{cc}
A(\alpha) & B^{T}(\alpha) \\
B(\alpha) & 0
\end{array}\right), \\
\tilde{L}_{\alpha \alpha} & :=L_{\alpha \alpha}+D_{1}^{-1} Z_{1}+D_{2}^{-1} Z_{2} .
\end{aligned}
$$

Denoting by $\tilde{S}^{-1}$ an approximate inverse of $S$ (approximate Stokes solver), an approximate inverse $\tilde{\mathcal{A}}^{-1}$ of the first diagonal block $\mathcal{A}$ of the condensed Hessian $K$ is given by

$$
\mathcal{A}=\left(\begin{array}{cc}
I(\alpha) & S \\
S & 0
\end{array}\right) \quad \Longrightarrow \quad \tilde{\mathcal{A}}^{-1}=\left(\begin{array}{cc}
0 & \tilde{S}^{-1} \\
\tilde{S}^{-1} & -\tilde{S}^{-1} I(\alpha) \tilde{S}^{-1}
\end{array}\right)
$$

Hence, a right-transform $K_{R}$ of $K$ can be obtained according to

$$
K_{R}:=\left(\begin{array}{cc|c}
I & 0 & -\tilde{S}^{-1} L_{\boldsymbol{\lambda}, \alpha} \\
0 & I & -\tilde{S}^{-1} L_{\mathbf{v}, \alpha}+\tilde{S}^{-1} I(\alpha) \tilde{S}^{-1} L_{\boldsymbol{\lambda}, \alpha} \\
-- & -- & ------------- \\
0 & 0 & I
\end{array}\right)
$$

The right transform $K_{R}$ provides a regular splitting of the condensed Hessian

$$
\begin{aligned}
& K K_{R}:=\left(\begin{array}{cc|c}
I(\alpha) & S & L_{\mathbf{v}, \alpha} \\
S & 0 & L_{\boldsymbol{\lambda}, \alpha} \\
-- & -- & -- \\
L_{\alpha, \mathbf{v}} & L_{\alpha, \boldsymbol{\lambda}} & \tilde{L}_{\alpha \alpha}
\end{array}\right)\left(\begin{array}{cc|c}
I & 0 & -\tilde{S}^{-1} L_{\boldsymbol{\lambda}, \alpha} \\
0 & I & -\tilde{S}^{-1} L_{\mathbf{v}, \alpha}+\tilde{S}^{-1} I(\alpha) \tilde{S}^{-1} L_{\boldsymbol{\lambda}, \alpha} \\
-- & -- & ------------ \\
0 & 0 & I
\end{array}\right) \\
& =\underbrace{\left(\begin{array}{cc|c}
I(\alpha) & S & 0 \\
S & 0 & 0 \\
-- & -- & -- \\
L_{\alpha, \mathbf{v}} & L_{\alpha, \boldsymbol{\lambda}} & \hat{L}_{\alpha \alpha}
\end{array}\right)}_{=: M_{1}}-\underbrace{\left(\begin{array}{cc|c}
0 & 0 & \left(I-S \tilde{S}^{-1}\right)\left(I(\alpha) \tilde{S}^{-1} L_{\boldsymbol{\lambda}, \alpha}-L_{\mathbf{v}, \alpha}\right) \\
0 & 0 & \left(S \tilde{S}^{-1}-I\right) L_{\boldsymbol{\lambda}, \alpha} \\
-- & -- & -------------- \\
0 & 0 & 0
\end{array}\right)}_{=: M_{2} \approx 0} .
\end{aligned}
$$

where

$$
\hat{L}_{\alpha \alpha}:=\tilde{L}_{\alpha \alpha}+L_{\alpha, \boldsymbol{\lambda}} \tilde{S}^{-1} I(\alpha) \tilde{S}^{-1} L_{\boldsymbol{\lambda}, \alpha}-L_{\alpha, \mathbf{v}} \tilde{S}^{-1} L_{\boldsymbol{\lambda}, \alpha}-L_{\alpha, \boldsymbol{\lambda}} \tilde{S}^{-1} L_{\mathbf{v}, \alpha}
$$


Let $\Delta \varphi^{*}:=\left(\Delta \mathbf{v}^{*}, \Delta \boldsymbol{\lambda}^{*}, \Delta \alpha^{*}\right)^{T}$ be the solution of $K \Delta \varphi^{*}=d$. Then, there holds

$$
K \Delta \varphi^{*}=d \Longleftrightarrow \underbrace{K K_{R}}_{=M_{1}-M_{2}} K_{R}^{-1} \Delta \varphi^{*}=d .
$$

The regular splitting $K K_{R}=M_{1}-M_{2}$ induces the iterative scheme

$$
K_{R}^{-1} \delta \varphi^{(\nu+1)}=K_{R}^{-1} \delta \varphi^{(\nu)}+M_{1}^{-1}\left(d-K \delta \varphi^{(\nu)}\right),
$$

which results in the right transforming iteration

$$
\delta \varphi^{(\nu+1)}=\delta \varphi^{(\nu)}+K_{R} M_{1}^{-1}\left(d-K \delta \varphi^{(\nu)}\right) .
$$

Let $\delta \varphi^{(0)}:=\left(\delta \mathbf{v}^{(0)}, \delta \boldsymbol{\lambda}^{(0)}, \delta \alpha^{(0)}\right)^{T}$ be given and compute $\delta \varphi^{(\ell+1)}, k \geq 0$, by

$$
\delta \varphi^{(\ell+1)}=\delta \varphi^{(\ell)}+K_{R} M_{1}^{-1}\left(d-K \delta \varphi^{(\ell)}\right) .
$$

Step 1: Compute the residual $r:=d-K \delta \varphi^{(\ell)}$.

Step 2: Compute $\delta \psi=\left(\delta \psi_{1}, \delta \psi_{2}, \delta \psi_{3}\right)^{T}$ as the solution of

$$
M_{1} \delta \psi=\left(\begin{array}{cc:c}
I(\alpha) & S & 0 \\
S & 0 & 0 \\
-- & -- & -- \\
L_{\alpha, \mathbf{v}} & L_{\alpha, \boldsymbol{\lambda}} & \hat{L}_{\alpha \alpha}
\end{array}\right)\left(\begin{array}{c}
\delta \psi_{1} \\
\delta \psi_{2} \\
-- \\
\delta \psi_{3}
\end{array}\right)=\left(\begin{array}{c}
r_{1} \\
r_{2} \\
-- \\
r_{3}
\end{array}\right)
$$

Step 2.1: Compute $\left(\delta \psi_{1}, \delta \psi_{2}\right)^{T}$ according to

$$
\left(\begin{array}{l}
\delta \psi_{1} \\
\delta \psi_{2}
\end{array}\right)=\left(\begin{array}{cc}
I(\alpha) & S \\
S & 0
\end{array}\right)^{-1}\left(\begin{array}{c}
r_{1} \\
r_{2}
\end{array}\right) \approx\left(\begin{array}{cc}
0 & \tilde{S}^{-1} \\
\tilde{S}^{-1} & -\tilde{S}^{-1} I(\alpha) \tilde{S}^{-1}
\end{array}\right)\left(\begin{array}{l}
r_{1} \\
r_{2}
\end{array}\right) .
$$

Step 2.2: Compute $\delta \psi_{3}$ as the solution of

$$
\hat{L}_{\alpha \alpha} \delta \psi_{3}=r_{3}-L_{\alpha, \mathbf{v}} \delta \psi_{1}-L_{\alpha, \boldsymbol{\lambda}} \delta \psi_{2} .
$$

As a termination criterion for the inexact Newton solves, we stop the inner iterations, if

$$
\theta_{\ell}:=\frac{1-\bar{\delta}_{\ell+1}}{1+\bar{\delta}_{\ell}} \frac{\left\|K_{R}^{-1} \delta \varphi^{(\ell+1)}\right\|}{\left\|K_{R}^{-1} \delta \varphi^{(\ell)}\right\|} \leq \frac{1}{4}
$$

where

$$
\bar{\delta}_{\ell+1}:=\frac{\sqrt{\varepsilon_{\ell}}}{\left\|\delta \varphi^{(\ell)}\right\|}
$$

with $\varepsilon_{\ell}$ being a lower bound for $\left\|\Delta \varphi-\delta \varphi^{(\ell)}\right\|$. Note that

$$
K_{R}^{-1} \delta \varphi^{(\ell)}=\left(\begin{array}{cc:c}
I & 0 & \tilde{S}^{-1} L_{\boldsymbol{\lambda}, \alpha} \\
0 & I & \tilde{S}^{-1} L_{\mathbf{v}, \alpha}-\tilde{S}^{-1} I(\alpha) \tilde{S}^{-1} L_{\boldsymbol{\lambda}, \alpha} \\
-- & -- & -------- \\
\hline 0 & 0 & I
\end{array}\right)\left(\begin{array}{c}
\delta \mathbf{v}^{(\ell)} \\
\delta \boldsymbol{\lambda}^{(\ell)} \\
--- \\
\delta d^{(\ell)}
\end{array}\right) .
$$




\section{Numerical results}

In the numerical examples, we consider Stokes flow with viscosity $\nu$ in channels $\Omega(\alpha) \subset \mathbb{R}^{2}$ with reference domain $\hat{\Omega}:=\Omega\left(\alpha^{0}\right)$ whose boundaries $\Gamma$ consist of the inflow and outflow boundaries $\Gamma_{\text {in }}, \Gamma_{\text {out }}$ and the lateral boundaries $\Gamma_{t o p}, \Gamma_{b o t}$ (cf. Fig. 1). The inflow $u_{\text {in }}$ on the inflow boundary $\Gamma_{i n}$ and the desired profiles $\mathbf{u}^{\mathbf{d}}, p^{d}$ as well as the weighting factors $\lambda_{i}, 1 \leq i \leq 2$, are given. We assume $m_{1}$ Bézier control points $\alpha_{i}, 1 \leq i \leq m_{1}$, on $\hat{\Gamma}_{\text {top }}$ and $m_{2}=m-m_{1}$ Bézier control points $\alpha_{i}, m_{1}+1 \leq i \leq m$, on $\hat{\Gamma}_{b o t}$ with given lower and upper bounds $\alpha_{i}^{\min }, \alpha_{i}^{\max }, 1 \leq i \leq m$. We use a discretization by Taylor-Hood P2/P1 elements [11] with respect to a simplicial triangulation $\mathcal{T}_{h}(\hat{\Omega})$ of the reference domain $\hat{\Omega}$ with $n_{1}$ degrees of freedom (DOF) for each velocity component and $n_{2}$ DOF for the pressure. The derivatives occurring in the KKT conditions and the Hessians have been computed by automatic differentiation [23]. We further refer to $k \in \mathbb{N}$ and $\ell_{k} \in \mathbb{N}$ as the numbers of the actual continuation step and the inexact Newton iteration, respectively. Moreover, $\mu$ and $\Delta \mu$ denote the barrier parameter and its increment, $\Theta$ stands for the parameter in the monotonicity test (cf. (3.12)), $\|\Delta \alpha\|_{2}$ and $\left\|\alpha-\alpha^{*}\right\|_{\infty}$ are the Euclidean norm of the Newton increment in the design variables and the maximum norm of the error in the design variables (in case the optimal design $\alpha^{*}$ is explicitly known), and $J$ is the value of the objective functional at the $k$-th continuation step. Finally, tolmin and tolnt refer to the termination criteria of the continuation process and the inexact Newton solves, respectively, and $h_{\max }$ denotes the maximal mesh width.

Example 1: The data of the first example are as follows

$$
\begin{aligned}
& \hat{\Omega}=\Omega\left(\alpha^{0}\right)=(1,5) \times(-1,+1) \text { with } \alpha^{0}=\left(+1,+1,+1,+1,-1,-1,-1,-1^{T},\right. \\
& \mathbf{u}^{d}, p^{d} \text { according to } \bar{\alpha}=(1.00,1.28,1.40,1.24,-1.00,-1.05,-1.20,-1.13)^{T}, \\
& \nu=1.0, u_{\text {in }}\left(x_{1}, x_{2}\right)=\left(6\left(1+x_{2}\right)\left(1-x_{2}\right), 0\right), \\
& \alpha^{\text {min }}=(0,0,0,0,-5,-5,-5,-5)^{T}, \alpha^{\text {max }}=(5,5,5,5,0,0,0,0)^{T} .
\end{aligned}
$$

The optimal solution $\left(\mathbf{u}^{*}, p^{*}, \alpha^{*}\right)$ has been chosen as

$$
\mathbf{u}^{*}=\mathbf{u}^{d} \quad, \quad p^{*}=p^{d} \quad, \quad \alpha^{*}=\bar{\alpha},
$$

where $\bar{\alpha}$ is the design associated with $\mathbf{u}^{d}, p^{d}$. Hence, the value $J^{*}$ of the objective functional

\begin{tabular}{|c|c|c|c|c|c|c|c|c|}
\hline$k$ & $\mu$ & $\Delta \mu$ & corr. & $\ell_{k}$ & $\Delta \alpha \|_{2}$ & $\mid \alpha-\alpha^{*} \|_{\infty}$ & $J$ & $\bar{\Theta}$ \\
\hline 0 & 100.0 & $(200.0)$ & - & - & - & $4.0 \mathrm{e}-01$ & $1.8 \mathrm{e}+00$ & - \\
\hline 1 & 100.0 & 163.3 & no & 1 & $6.9 \mathrm{e}-01$ & $1.4 \mathrm{e}-03$ & $1.1 \mathrm{e}-05$ & 0.36 \\
\hline & & & & 2 & $6.0 \mathrm{e}-06$ & $1.4 \mathrm{e}-03$ & $1.1 \mathrm{e}-05$ & 0.33 \\
\hline 2 & 263.3 & 459.8 & no & 1 & $1.9 \mathrm{e}-03$ & $5.0 \mathrm{e}-04$ & $1.5 \mathrm{e}-06$ & 0.03 \\
\hline 3 & 723.1 & - & - & - & - & $4.0 \mathrm{e}-04$ & $8.8 \mathrm{e}-07$ & - \\
\hline
\end{tabular}
at optimality is zero.

Table 1: Example 1: Discretization data and termination criteria

\begin{tabular}{|c|c|c|c|c|c|c|c|c|}
\hline $2 n_{1}$ & $n_{2}$ & $m_{1}$ & $m_{2}$ & $\mu^{0}$ & $\Delta \mu^{0}$ & tolnt & tolmin & $h_{\max }$ \\
\hline 4998 & 646 & 4 & 4 & 100 & 200 & $4.0 \mathrm{e}-03$ & $1.0 \mathrm{e}-06$ & 0.15 \\
\hline
\end{tabular}

Table 2: Example 1: Convergence history of the continuation method 

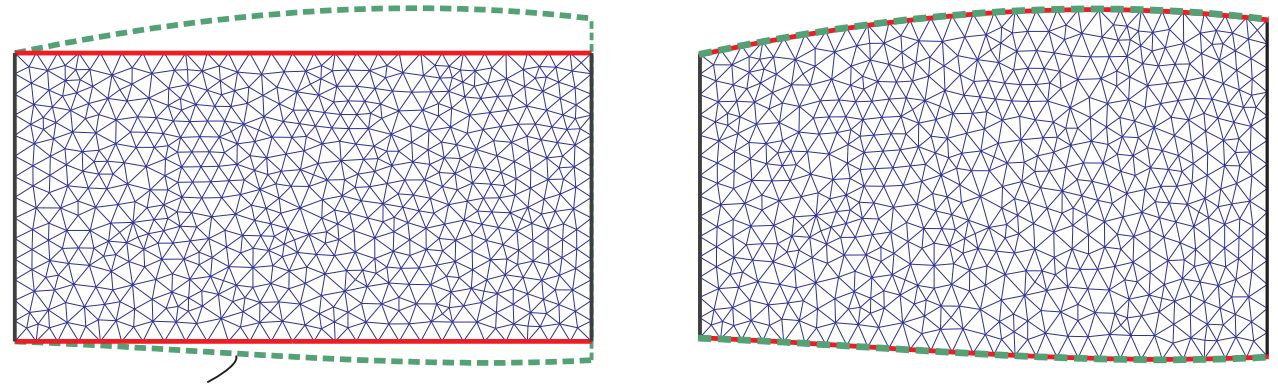

dotted line: optimal shape
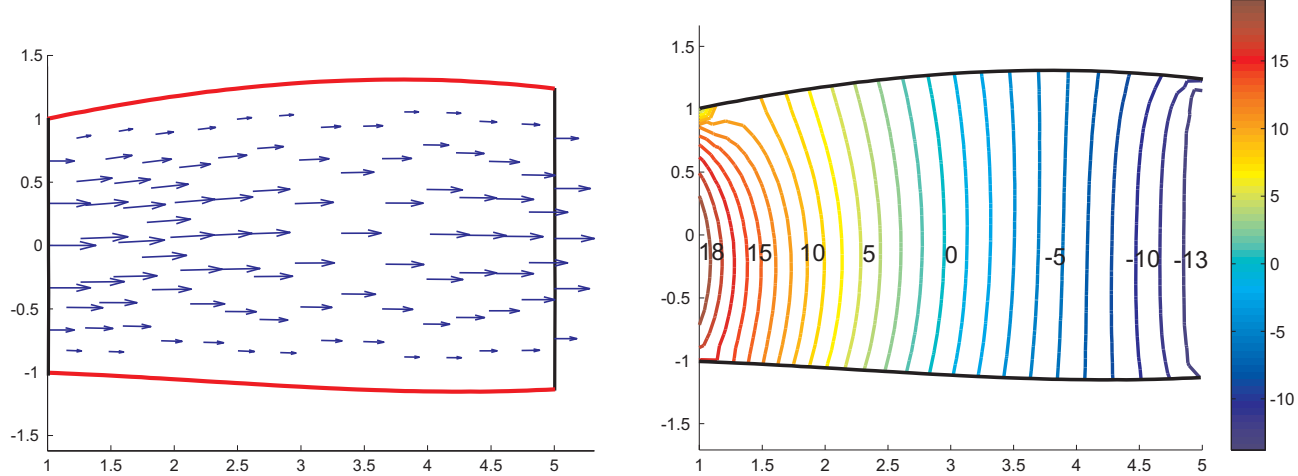

Figure 1: Results for Example 1: Initial shape (top left), optimal shape after the third iteration (top right), the optimal velocity field as an arrow plot (bottom left) and the $L_{0^{-}}^{2}$ gauged pressure field shown by equipotential lines (bottom right).

The data of the discretization, the initial value $\mu^{0}$ of the inverse of the barrier parameter and its initial increment $\Delta \mu^{0}$ as well as the termination criteria tolnt and tolmin are shown in Table 1. Table 2 contains the convergence history of the adaptive path-following primaldual interior-point method. The algorithm was stopped by criterion tolmin after three continuation steps and a computational time of 55 minutes (AMD Athlon, $1.8 \mathrm{GHz}$ ) with the final design vector

$$
\alpha^{3}=(1.0000,1.2797,1.4001,1.2399,-1.0000,-1.0496,-1.2000,-1.1297)^{T} .
$$

Due to our experience, a fast convergence of the algorithm requires initial parameters $\mu^{0}$ and $\Delta \mu^{0}$ that are not chosen too small. In particular, for too small $\Delta \mu^{0}$ the algorithm starts too ,,carefully" and does not achieve satisfactory progress within a reasonable number of outer iterations.

Figure 1 displays the initial and optimal shape (top) and the associated velocity and pressure field (bottom).

Example 2: This example has been constructed in such a way that the desired velocity profile is outside the range of the admissible design variables. Consequently, the value $J^{*}$ of the objective functional at optimality is greater than zero (cf. Table 4). It can be observed that the upper computed Bézier curve tries to ,,mimic" the corresponding part of the shape associated with the desired velocity (cf. Figure 2, bottom right). The data for $\alpha^{\min }, \alpha^{\max }$, $\mathbf{u}_{i n}$ etc. are the same as in Example 1, except that the desired velocity field corresponds to the following vector of design variables

$$
\bar{\alpha}=(2.50,1.65,1.15,0.90,-1.30,-0.85,-1.20,-1.35)^{T} .
$$


After reaching the criterion $\mu>\mu_{\max }=15000$, the computed optimal design turned out to be

$$
\alpha^{8}=(1.0000,0.5171,0.0928,0.0000,-1.0000,-0.6795,-1.2788,-1.0710)^{T} .
$$

Table 3: Example 2: Discretization data and termination criteria

\begin{tabular}{|c|c|c|c|c|c|c|c|c|}
\hline $2 n_{1}$ & $n_{2}$ & $m_{1}$ & $m_{2}$ & $\mu^{0}$ & $\Delta \mu^{0}$ & tolnt & tolmin & $h_{\max }$ \\
\hline 2762 & 361 & 4 & 4 & 100 & 300 & $4.0 \mathrm{e}-03$ & $1.0 \mathrm{e}-06$ & 0.20 \\
\hline
\end{tabular}

Table 4: Example 2: Convergence history of the continuation method

\begin{tabular}{|c|c|c|c|c|c|c|c|}
\hline$k$ & $\mu$ & $\Delta \mu$ & corr. & $\ell_{k}$ & $\|\Delta \alpha\|_{2}$ & $J$ & $\bar{\Theta}$ \\
\hline 0 & 100.0 & $(300.0)$ & $\begin{array}{llll}- & \end{array}$ & - & - & $2.4 \mathrm{e}+01$ & $\begin{array}{lll}- & \end{array}$ \\
\hline \multirow[t]{4}{*}{1} & 100.0 & 269.6 & no & 1 & $1.8 \mathrm{e}+00$ & $4.0 \mathrm{e}+00$ & 0.30 \\
\hline & & & & 2 & $1.2 \mathrm{e}-02$ & $3.9 \mathrm{e}+00$ & 0.98 \\
\hline & & & & 3 & $2.3 \mathrm{e}-01$ & $1.9 \mathrm{e}+00$ & 0.51 \\
\hline & & & & 4 & $1.7 \mathrm{e}-01$ & $7.0 \mathrm{e}-01$ & 0.25 \\
\hline \multirow[t]{2}{*}{2} & 369.6 & 274.3 & no & 1 & $3.1 \mathrm{e}-02$ & $6.8 \mathrm{e}-01$ & 0.17 \\
\hline & & & & 2 & $5.8 \mathrm{e}-03$ & $6.7 \mathrm{e}-01$ & 0.24 \\
\hline 3 & 643.8 & 512.2 & no & 1 & $1.3 \mathrm{e}-03$ & $6.7 \mathrm{e}-01$ & 0.06 \\
\hline 4 & 1156.0 & 1321.6 & no & 1 & $7.4 \mathrm{e}-04$ & $6.7 \mathrm{e}-01$ & 0.03 \\
\hline 5 & 2477.6 & 3205.5 & no & 1 & $7.2 \mathrm{e}-04$ & $6.7 \mathrm{e}-01$ & 0.04 \\
\hline 6 & 5683.1 & 1584.6 & no & 1 & $4.1 \mathrm{e}-04$ & $6.7 \mathrm{e}-01$ & 0.85 \\
\hline 7 & 7267.7 & 9043.7 & no & 1 & $2.1 \mathrm{e}-04$ & $6.7 \mathrm{e}-01$ & 0.01 \\
\hline 8 & 16311.4 & $(8077.4)$ & no & 1 & $1.3 \mathrm{e}-04$ & $6.7 \mathrm{e}-01$ & 0.54 \\
\hline
\end{tabular}

Example 3: In this example, we consider a channel with a backward facing step (cf. Figure 3, top left). Here, the three horizontal segments of the geometry are fixed and only the segment that connects the two lower horizontal segments is variable. In this setting, the function that describes the bottom segment of the geometry is a composite Bézier curve consisting of three curves of degree 0,4 and 0 , respectively. Note that the composite curve is only continuous. The data for this geometry are as follows

$$
\begin{aligned}
& \hat{\Omega}=\Omega\left(\alpha^{0}\right) \text { with } \alpha^{0}=(+1.0,-0.5,-0.5,-1.5,-1.5,-1.5)^{T}, \\
& \mathbf{u}^{d}, p^{d} \text { according to } \bar{\alpha}=(+1.0,-0.5,-0.55,-0.6,-0.6,-1.5)^{T}, \\
& \nu=1.0, u_{i n}\left(x_{1}, x_{2}\right)=\left(6\left(1+x_{2}\right)\left(1-x_{2}\right), 0\right), \\
& \alpha^{\text {min }}=(0,-5,-5,-5,-5,-5)^{T}, \alpha^{\text {max }}=(5,0,0,0,0,0)^{T} .
\end{aligned}
$$

As in Example 1, the optimal solution $\left(\mathbf{u}^{*}, p^{*}, \alpha^{*}\right)$ has been chosen as $\mathbf{u}^{*}=\mathbf{u}^{d}, p^{*}=p^{d}$ and $\alpha^{*}=\bar{\alpha}$. Table 5 provides information about the discretization and Table 6 displays the convergence history of the adaptive path-following algorithm.

Table 5: Example 3: Discretization data and termination criteria

\begin{tabular}{|c|c|c|c|c|c|c|c|}
\hline $2 n_{1}$ & $n_{2}$ & $m_{1}$ & $m_{2}$ & $\mu^{0}$ & $\Delta \mu^{0}$ & tolnt & tolmin \\
\hline 2856 & 191 & 1 & 5 & 100 & 300 & $4.0 \mathrm{e}-03$ & $1.0 \mathrm{e}-06$ \\
\hline
\end{tabular}



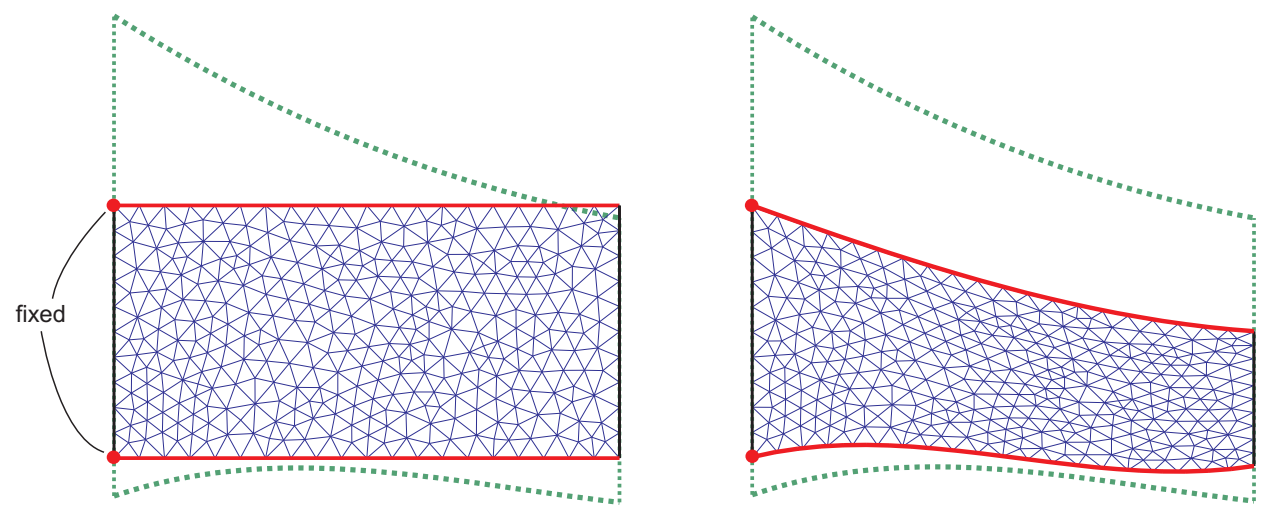

Figure 2: Results for Example 2: Initial shape (left) and the optimal shape after 8 iterations (right). Here, the first Bézier point of each curve (top and bottom) of the initial shape are fixed and different from the corresponding points of the shape corresponding to the desired velocity profile (dotted line).
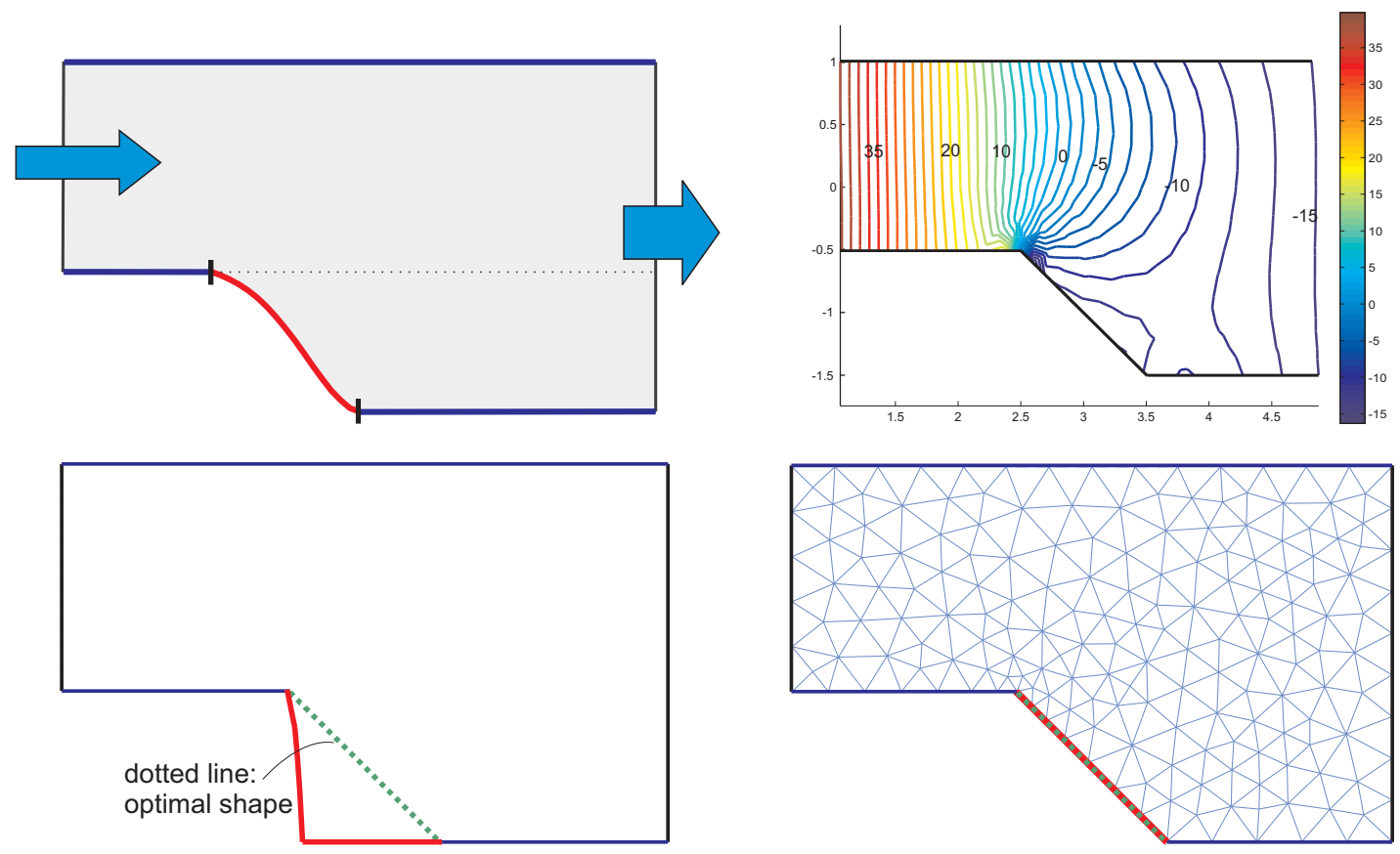

Figure 3: Results for Example 3: A scheme of the backward step geometry (top left) along with the optimal pressure filed (top right), initial and optimal shape after 7 outer iterations (bottom left and right). 
Table 6: Example 3: Convergence history of the continuation method

\begin{tabular}{|c|c|c|c|c|c|c|c|c|}
\hline$k$ & $\mu$ & $\Delta \mu$ & corr. & $\ell_{k}$ & $\mid \Delta \alpha \|_{2}$ & $\left\|\alpha-\alpha^{*}\right\|_{\infty}$ & $J$ & $\Theta$ \\
\hline 0 & 100.0 & $(300.0)$ & - & - & - & $9.0 \mathrm{e}-01$ & $2.6 \mathrm{e}+00$ & - \\
\hline 1 & 100.0 & $(300.0)$ & yes & 1 & $1.2 \mathrm{e}+00$ & $1.7 \mathrm{e}-01$ & $9.6 \mathrm{e}-01$ & 0.58 \\
\hline & & & & 2 & $8.8 \mathrm{e}-01$ & $1.7 \mathrm{e}-01$ & $1.3 \mathrm{e}-01$ & 618.42 \\
\hline 1 & 100.0 & 425.5 & no & 1 & $2.1 \mathrm{e}-01$ & $3.3 \mathrm{e}-02$ & $4.3 \mathrm{e}-04$ & 0.11 \\
\hline 2 & 525.5 & 417.1 & no & 1 & $1.2 \mathrm{e}-01$ & $3.3 \mathrm{e}-02$ & $2.3 \mathrm{e}-03$ & 0.41 \\
\hline & & & & 2 & $3.3 \mathrm{e}-02$ & $2.5 \mathrm{e}-02$ & $2.3 \mathrm{e}-03$ & 0.58 \\
\hline & & & & 3 & $1.6 \mathrm{e}-02$ & $2.4 \mathrm{e}-02$ & $2.0 \mathrm{e}-03$ & 0.92 \\
\hline & & & & 4 & $2.0 \mathrm{e}-02$ & $2.9 \mathrm{e}-03$ & $1.6 \mathrm{e}-05$ & 0.43 \\
\hline & & & & 5 & $5.7 \mathrm{e}-04$ & $3.2 \mathrm{e}-03$ & $2.5 \mathrm{e}-05$ & - \\
\hline 3 & 942.6 & 323.5 & no & 1 & $2.9 \mathrm{e}-03$ & $3.6 \mathrm{e}-03$ & $5.1 \mathrm{e}-05$ & 0.34 \\
\hline 4 & 1266.1 & 283.7 & no & 1 & $1.4 \mathrm{e}-03$ & $3.5 \mathrm{e}-03$ & $4.9 \mathrm{e}-05$ & 0.27 \\
\hline 5 & 1549.8 & 593.1 & no & 1 & $1.7 \mathrm{e}-04$ & $2.9 \mathrm{e}-03$ & $3.3 \mathrm{e}-05$ & 0.05 \\
\hline 6 & 2142.9 & 2265.3 & no & 1 & $1.3 \mathrm{e}-04$ & $2.1 \mathrm{e}-03$ & $1.7 \mathrm{e}-05$ & 0.01 \\
\hline 7 & 4408.2 & 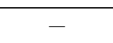 & - & - & - & $2.0 \mathrm{e}-04$ & $1.9 \mathrm{e}-07$ & - \\
\hline
\end{tabular}

\section{References}

[1] Allaire, G. (2002). Shape Optimization by the Homogenization Method. Springer, Berlin-Heidelberg-New York.

[2] Bendsøe, M.P. (1995). Optimization of Structural Topology, Shape, and Material. Springer, Berlin-Heidelberg-New York.

[3] Bendsøe, M.P., and Sigmund, O. (2003). Topology Optimization: Theory, Methods and Applications. Springer, Berlin-Heidelberg-New York.

[4] Biegler, L.T., Ghattas, O., Heinkenschloss, M., and van Bloemen Waanders, B. (eds.) (2003). Large-Scale PDE-Constrained Optimization. Lecture Notes in Computational Science and Engineering, Vol. 30, Springer-Verlag, Berlin, Heidelberg, New York.

[5] Biegler, L.T., Nocedal, J., and Schmid, C. (1995). A reduced Hessian method for large-scale constrained optimization. SIAM J. Optimization, 5, 314-347.

[6] Biros, G., and Ghattas, O. (2003). Inexactness issues in the Lagrange-NewtonKrylov-Schur method for PDE-constrained optimization. In: Large-scale PDEconstrained optimization, Santa Fe, NM, 2001, (Biegler, L.T., et al.; eds.), pp. 93-114, Lect. Notes Comput. Sci. Eng., Vol. 30, Springer,Berlin-Heidelberg-New York.

[7] Biros, G., and Ghattas, O. (2005). Parallel Lagrange-Newton-Krylov-Schur methods for PDE-constrained optimization. part $i$ : the Krylov-Schur solver. SIAM J. Sci. Comp. 27, 687-713.

[8] Biros, G., and Ghattas, O. (2005). Parallel Lagrange-Newton-Krylov-Schur methods for PDE-constrained optimization. part ii: the Lagrange-Newton solver and its application to optimal control of staedy viscous flows. SIAM J. Sci. Comp. 27, 714-739.

[9] Boggs, P.T., and Tolle, J.W. (1995). Sequential quadratic programming. Acta Numerica, 4, 1-50.

[10] Bonnans, J.F., Gilbert, J.C., Lemaréchal, C., and Sagastiz?'abal, C.A. (2003). Numerical Optimization. Theoretical and Practical Aspects. Springer, BerlinHeidelberg-New York.

[11] Brezzi, F., and Fortin, M. (1991). Mixed and Hybrid Finite Element Methods. Springer, Berlin-Heidelberg-New York. 
[12] Byrd, R.H., Hribar,M.E., and Nocedal, J. (1999). An interior point algorithm for large scale nonlinear programming. SIAM J. Optimization, 9, 877-900.

[13] Cherkaev, A. (2000). Variational Methods for Structural Optimization. Springer, New York.

[14] Delfour, M.C., and Zolesio, J.P. (2001). Shapes and Geometries: Analysis, Differential Calculus and Optimization. SIAM, Philadelphia.

[15] Dennis, J., Heinkenschloss, M., and Vicente, L.N. (1998). Trust-region interior-point $S Q P$ algorithms for a class of nonlinear programming problems. SIAM J. Control Optim., 36, 1750-1794.

[16] Deuflhard, P. (2004). Newton Methods for Nonlinear Problems. Affine Invariance and Adaptive Algorithms. Springer, Berlin-Heidelberg-New York.

[17] Forsgren, A., and Gill, Ph.E. (1998). Primal-dual interior methods for nonconvex nonlinear programming. SIAM J. Optimization, 8, 1132-1152.

[18] Forsgren, A., Gill, Ph.E. and Wright, M.H. (2002). Interior methods for nonlinear optimization. SIAM Review, 44, 525-597.

[19] Forsgren, A., Gill, Ph.E., and Shinnerl, J.R. (1996). Stability of symmetric illconditioned systems arising in interior methods for constrained optimization. SIAM J. on Matrix Analysis and Applications, 17, 35-46.

[20] Gay, D.M., Overton, M.I., and Wright, M.H. (1998). Primal-dual interior method for nonconvex nonlinear programming. In: Advances in Nonlinear Programming (Y. Yuan; ed.), pp. 31-56, Kluwer, Dordrecht.

[21] Ghattas, O., and Orozco, C.E. (1997). A parallel reduced Hessian SQP method for shape optimization. In: Multidisciplinary Design Optimization: State-of-the-Art (Alexandrov, N., and Hussaini, M. (eds.)), pp. 133-152, SIAM, Philadelphia.

[22] Gill, Ph.E., Murray, W., and Saunders, M.A. (2002). SNOPT: an SQP algorithm for large-scale constrained optimization. SIAM J. Optimization, 12, 979-1006.

[23] Griewank, A. (2000). Evaluating Derivatives, Principles and Techniques of Automatic Differentiation. SIAM, Phildelphia.

[24] Haslinger, J., and Neittaanmäki, P. (1988). Finite Element Approximation for Optimal Shape Design: Theory and Applications. John Wiley \& Sons, Chichester.

[25] Haslinger, J., and Mäkinen, R.A.E. (2004). Introduction to Shape Optimization: Theory, Approximation, and Computation. SIAM, Philadelphia.

[26] Heinkenschloss, M., and Vicente, L.N. (2001). Analysis of inexact trust-region interior-point algorithms. SIAM J. Optimization, 12, 283-302.

[27] Herskovits, J., Dias, G., Santos, G., and Mota Soares, C.M. (2000). Shape structural optimization with an interior point nonlinear programming algorithm. Struct. Multidisc. Optim., 20, 107-115.

[28] Hinton, E., and Sienz,J. (1996). Adaptive Finite Element Analysis and Shape Optimization. SaxeCoburg Publications, Edinburgh.

[29] Hoppe, R.H.W., Linsenmann, C., and Petrova, S.I. (2006). Primal-dual Newton methods in structural optimization. Comp. Visual. Sci. 9, 71-87.

[30] Hoppe, R.H.W., and Petrova, S.I. (2004). Primal-dual Newton interior point methods in shape and topology optimization. Numerical Linear Algebra with Applications 11, 413-429.

[31] Hoppe, R.H.W., Petrova, S.I., and Schulz, V. (2002). A primal-dual Newton-type interior-point method for topology optimization. Journal of Optimization Theory and Applications 114, 545-571. 
[32] Maar, B., and Schulz, V. (2000). Interior point methods in topology optimization. Structural Optimization, 19, 214-224.

[33] Mohammadi, B. and Pironneau, O. (2001). Applied Shape Optimization for Fluids. Oxford University Press, Oxford.

[34] Nocedal, J., and Wright, S.J. (1999). Numerical Optimization. Springer, New York.

[35] Pironneau, O. (1984). Optimal Shape Design for Elliptic Systems. Springer, BerlinHeidelberg-New York.

[36] Rozvany, G. (1989). Structural Design via Optimality Criteria. Kluwer, Dordrecht.

[37] Sachs, E.W., and Volkwein, S. (2002). Augmented Lagrange-SQP methods with Lipschitz-continuous Lagrange multiplier updates. SIAM J. Numer. Anal., 40, 233253.

[38] Schulz, V. (2004). Simultaneous solution approaches for large optimization problems. J. Comput. Appl. Math., 164/165, 629-641.

[39] Sokolowski, J., and Zolesio, J.P. (1992). Introduction to Shape Optimization. Springer, Berlin-Heidelberg-New York, 1992.

[40] Ulbrich, M., and Ulbrich, S. (2000). Superlinear convergence of affine-scaling interior-point Newton methods for infinite-dimensional nonlinear problems with pointwise bounds. SIAM J. Control Optim., 38, 1938-1984.

[41] Ulbrich, M., Ulbrich, S., and Heinkenschloss, M. (1999). Global convergence of trustregion interior-point algorithms for infinite-dimensional nonconvex minimization subject to pointwise bounds. SIAM J. Control Optimization, 37, 731-764.

[42] Wittum, G. (1989). On the convergence of multigrid iterations with transforming smoothers. Theory with applications to the Navier-Stokes equations. Numer. Math., $\mathbf{5 7}, 15-38$.

[43] Wright, M.H. (1992). Interior methods for constrained optimization. Acta Numerica, 1, 341-407.

[44] Wright, S.J. (1997). Primal-Dual Interior-Point Methods. SIAM, Philadelphia.

[45] Ye, Y. (1997). Interior-Point Algorithm: Theory and Analysis. Wiley, New York. 